2. Gottlieb, et al. JAMA 2021;325:632-44.

3. Garibaldi, et al. JAMA NetwOpen 2021:4:e213071.

4. Beigel, et al. N Engl J Med 2020;383:1813-26.

5. Kalil, et al. N Engl J Med 2020.

6. Temesgen, et al. medRxiv 2021.

\section{S52 CONVENTIONAL OXYGEN THERAPY VERSUS CPAP AS A CEILING OF CARE IN WARD-BASED PATIENTS WITH COVID-19: A MULTI-CENTRE COHORT EVALUATION} ${ }^{6} \mathrm{~A}$ Ashish, ${ }^{1} \mathrm{~A}$ Bentley, ${ }^{7} \mathrm{~T}$ Bongers, ${ }^{8} \mathrm{~T}$ Gatheral, ${ }^{1} \mathrm{TW}$ Felton, ${ }^{1} \mathrm{~N}$ Chaudhuri, ${ }^{1} \mathrm{~L}$ Pearmain. ${ }^{1}$ North West Lung centre, Wythenshawe Hospital, Manchester University NHS Foundation Trust, Manchester, UK; ${ }^{2}$ North Manchester General Hospital, Manchester University NHS Foundation Trust, Manchester, UK; ${ }^{3}$ Research and Development, Blackpool Teaching Hospitals NHS Foundation Trust, Blackpool, UK; ${ }^{4}$ NWCORR North West Collaborative Organisation for Respiratory Research study group, North West, UK; ${ }^{5}$ Respiratory Department, Royal Preston Hospital, Lancashire Teaching Hospitals NHS Foundation Trust, Preston, UK; ${ }^{6}$ Respiratory Department, Royal Albert Edward Infirmary, Wrightington, Wigan and Leigh Teaching Hospitals NHS Foundation Trust, Wigan, UK; ${ }^{7}$ Respiratory Department, Blackpool Teaching Hospitals NHS Foundation Trust, Blackpool, UK; ${ }^{8}$ Respiratory department, University Hospitals of Morecambe Bay NHS Foundation Trust, Lancaster, UK

\subsection{6/thorax-2021-BTSabstracts.58}

Background Continuous positive airway pressure (CPAP) therapy is commonly used for respiratory failure due to severe COVID-19 pneumonitis, including in patients deemed unlikely to benefit from invasive mechanical ventilation (nIMV). Little evidence exists demonstrating superiority over conventional oxygen therapy, as acknowledged by current pragmatic guidelines, whilst ward-level delivery of CPAP presents practical challenges. Precedent studies have been limited by small numbers, subjective physician treatment-selection, and lack of a control group. We sought to compare clinical outcomes of oxygen therapy versus CPAP therapy in patients with COVID19 who were nIMV.

Methods The North West Collaborative Organisation for Respiratory Research (NWCORR), a trainee-led network, performed a retrospective multi-centre cohort evaluation. Patient inclusion criteria were: a clinical diagnosis of COVID-19, a treatment escalation plan of ward-level care, treatment at a

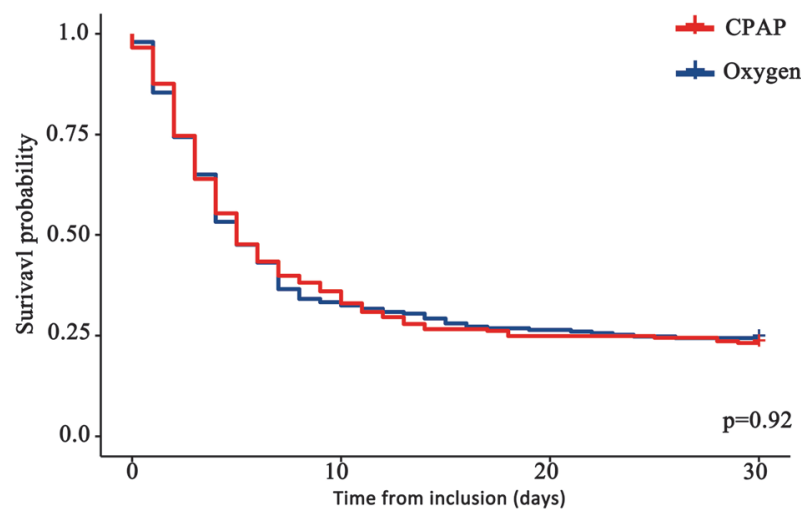

\begin{tabular}{|c|c|c|c|c|}
\hline \multicolumn{5}{|c|}{ Number at risk } \\
\hline Oxygen & 246 & 82 & 65 & 60 \\
\hline CPAP & 233 & 84 & 58 & 54 \\
\hline & 0 & 10 & 20 & 30 \\
\hline
\end{tabular}

Abstract S52 Figure 1 Kaplan-Meier curve comparing overall survival in the two treatment groups (conventional oxygen therapy vs continuous positive airway pressure therapy). The null hypothesis of no survival difference is evaluated with a log-rank test $(p=0.92)$ hospital only providing one respiratory support strategy and clinical frailty score $\leq 6$. Patients were cohorted according to respiratory support strategy, either being CPAP in accordance with national guidelines or oxygen therapy requiring $\mathrm{FiO}_{2}$ $\geq 0.4$ for over 12 hours; who would therefore have been eligible for CPAP if treated at a CPAP cohort hospital. Logistic regression modelling, using generalised estimating equations to account for within-hospital clustering, was performed to compare 30-day mortality between treatment groups, accounting for important confounders.

Results Seven hospitals provided data for 479 patients during the UK COVID-19 pandemic in 2020. Overall 30-day mortality was $75.6 \%$ in the oxygen group (186/246 patients) and $77.7 \%$ in the CPAP group (181/233 patients) (figure 1). A lack of evidence for a treatment effect persisted in the adjusted model (adjusted 30-day mortality odds ratio comparing CPAP to oxygen of $0.84,95 \%$ CI $0.57-1.23, \mathrm{p}=0.37$ ). $49.8 \%$ of patients receiving CPAP-therapy (118/237) chose to discontinue it.

Discussion This is, as far as we are aware, the first study comparing conventional oxygen therapy with CPAP in cohorts unaffected by physician selection. No survival difference was found between using oxygen alone or CPAP to treat patients with severe COVID-19 who were nIMV. A high patient-initiated discontinuation rate for CPAP suggests a significant treatment burden. Further reflection is warranted on the continued widespread use of CPAP in this patient group.

Please refer to page A189 for declarations of interest related to this abstract.

\section{S53 IMPACT OF EMPIRICAL ANTIBIOTIC USE IN PATIENTS WITH COVID-19 ON MORBIDITY AND MORTALITY DURING THE FIRST AND SECOND UK SARS-COV-2 WAVES}

S Waring, G Gamtkitsulashvili, S Kumar, Y Narayan, A D'Souza, S Jiwani, O Taylor, G Collins, K Patrick, A Sethuraman, S Naik, S Kuckreja, R Ragatha, M Anwar, U Ekeowa, P Russell. The Princess Alexandra Hospital, Harlow, Essex, UK

\subsection{6/thorax-2021-BTSabstracts.59}

Background Poor antimicrobial stewardship is frequently observed in COVID-19 patients and relates to greater mortality when empirical antibiotics are administered without compelling evidence of bacterial co-infection. ${ }^{1}$ Here, we assess the impact of antibiotic administration in COVID-19 patients on inpatient morbidity and mortality during the first and second UK SARS-CoV-2 waves.

Methods Two representative four-month timeframes of RTPCR positive COVID-19 admissions to a Greater London District General Hospital were retrospectively analysed, with 481 patients between 15th February 2020 and 15th June 2020 representing the first wave, and 1342 between $1^{\text {st }}$ November 2020 and 28th February 2021 for the second wave. Morbidity was measured by mean length-of-stay. Independent correlation was assessed with multilinear regression analysis adjusting for demographics and comorbidities.

Results $87.9 \%$ of first and $86.0 \%$ of second wave inpatients received at least one antibiotic, despite only $21.4 \%$ and $16.8 \%$ showing bacterial culture positivity of a non-contaminant pathogen, respectively. A mean 2.41 and 2.05 antibiotic types were administered per patient during first and second COVID-19 waves. Antibiotic administration was independently 


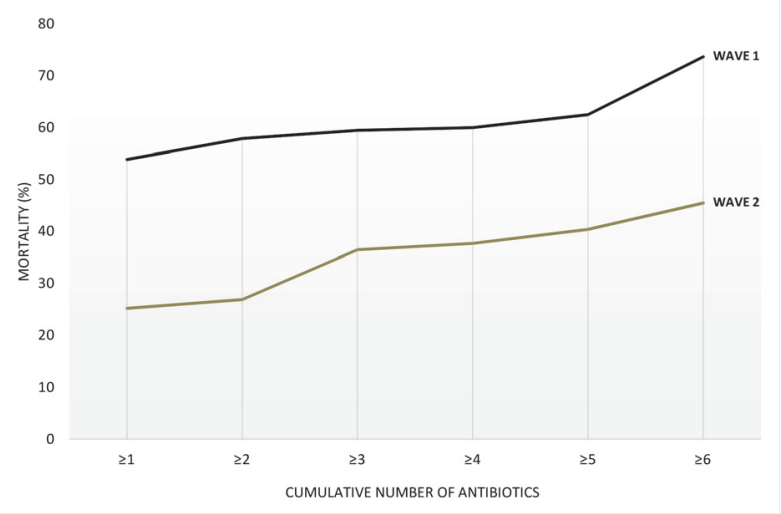

Abstract S53 Figure 1 Rates of mortality against cumulative number of antibiotics received per patient during inpatient spell.

associated with increased inpatient mortality in both the first wave $(54.1 \%$ mortality for $\geq 1$ antibiotic vs $19.0 \%$ receiving no antibiotic, $\mathrm{p}<0.00001)$ and second wave $(25.2 \%$ mortality for $\geq 1$ antibiotic vs $4.3 \%$ receiving no antibiotic, $\mathrm{p}<0.00001)$. Successive numbers of antibiotics related to progressive worsening of mortality rates in both waves (OR $1.453, \mathrm{p}<0.0001)$. Antibiotic use was also associated with prolonged length-of-stay in the first wave (13.0 \pm 13.4 days for $\geq 1$ antibiotic vs $6.9 \pm 12.1$ receiving no antibiotic, $p=0.0011$ ) and second wave $(11.9 \pm 11.7$ days for $\geq 1$ antibiotic vs 6.6 \pm 10.6 receiving no antibiotic, $\mathrm{p}=0.00005)$.

Conclusion In both COVID-19 waves, antibiotic administration correlated to increased inpatient morbidity and mortality. Given a near-linear relationship of mortality and cumulative antibiotic numbers, antimicrobial stewardship is essential, and tapering an appropriate therapy for likely responsible pathogens will yield lower mortality compared to overlapping coverage and inappropriate escalation. We strongly discourage the use of empirical antibiotics without supporting biochemical evidence of bacterial co-infection for possible future COVID19 waves.

\section{REFERENCE}

1. Russell C, et al. Lancet Microbe. 2021 Jun 2. https://doi.org/10.1016/S2666-5247 (21)00090-2

\section{Understanding COVID-19 mechanisms}

\section{S54 ELEVATED NETOSIS AND MIGRATION BUT IMPAIRED ANTI-MICROBIAL RESPONSES IN NEUTROPHILS FROM NON-ICU, HOSPITALIZED COVID-19 PATIENTS}

K Belchamber, S Thein, J Hazeldine, F Grudzinska, A Jasper, M Hughes, KP Yip, E Sapey, D Parekh, D Thickett, A Scott. University of Birmingham, Birmingham, UK

\subsection{6/thorax-2021-BTSabstracts.60}

Rational Infection with the SARS-CoV2 virus is associated with elevated neutrophil counts. Evidence of neutrophil dysfunction in COVID-19 is based predominantly on transcriptomics or single functional assays. Cell functions are interwoven pathways, and so understanding the effect of COVID-19 across the spectrum of neutrophil function may identify therapeutic targets to treat disease.

Objectives Examine neutrophil phenotype and functional capacity in COVID-19 patients versus age-matched controls (AMC).
Methods Isolated neutrophils from 41 non-ICU COVID-19 patients and 23 AMC underwent ex vivo analyses for migration, phagocytosis of Streptococcus pneumoniae, reactive oxygen species (ROS) generation, neutrophil extracellular trap formation (NETosis) and cell surface receptor expression. Serum DNAse 1 activity was measured, alongside circulating levels of cell-free (cf)DNA, myeloperoxidase (MPO), VEGF, IL-6 and sTNFRI. All measurements were correlated to clinical outcome. Serial sampling on day 3-5 post hospitalisation were also measured.

Results Compared to AMC, COVID-19 neutrophils demonstrated elevated transmigration $(p=0.0397)$ and NETosis $(\mathrm{p}=0.0366)$, but impaired phagocytosis $(\mathrm{p}=0.0236)$ associated with impaired ROS generation $(\mathrm{p}<0.0001)$. Surface expression of CD54 $(\mathrm{p}<0.0001)$ and CD11c $(\mathrm{p}=0.0008)$ was significantly increased and CD11b significantly decreased $(p=0.0229)$ on COVID-19 patient neutrophils. On day 3-5 follow-up, levels of senescent neutrophils increased compared to day 1 (indicated by decreased CXCR2 and elevated CXCR4 expression $(p=0.0332))$. COVID-19 patients showed increased systemic markers of NETosis including increased cfDNA $(p=0.0153)$ and impaired DNAse activity $(\mathrm{p}<0.0 .001)$. MPO, VEGF, sTNFRI, and IL-6 $(\mathrm{p}<0001)$ were elevated in COVID-19, which positively correlated with disease severity by $4 \mathrm{C}$ score. Conclusion COVID-19 is associated with neutrophil dysfunction across all main effector functions, with altered phenotype, elevated migration, impaired antimicrobial responses and elevated NETosis. These changes represent a clear mechanism for tissue damage and highlight that targeting neutrophil function may help modulate COVID-19 severity.

Please refer to page A189 for declarations of interest related to this abstract.

\section{\begin{tabular}{l|l} 
S55 & PERSISTENT CHANGES TO THE NASAL CILIATED
\end{tabular} EPITHELIUM FOLLOWING SARS-COV2 INFECTION: A LONGITUDINAL COHORT ANALYSIS FROM FOLLOW- COVID}

${ }^{1} \mathrm{~A}$ Shoemark, ${ }^{1} \mathrm{M}$ Bottier, ${ }^{2} \mathrm{~A}$ Pinto, 'L Delgado, ${ }^{1} \mathrm{H}$ Abo-Leyah, ${ }^{2} \mathrm{R}$ Rai, ${ }^{1} \mathrm{~B}$ Parcell, ${ }^{1} \mathrm{C}$ Hocking, ${ }^{2} \mathrm{C}$ Hogg, ${ }^{1} \mathrm{~A}$ Dicker, ${ }^{1} \mathrm{~F}$ Khan, ${ }^{1} \mathrm{~S}$ Gallant, 'DM Cassidy, ${ }^{1} \mathrm{D}$ Connell, ${ }^{1} \mathrm{JD}$ Chalmers. 'University of Dundee, Dundee, UK; ${ }^{2}$ Royal Brompton Hospital, London, UK

\subsection{6/thorax-2021-BTSabstracts.61}

Background SARS-CoV2 binds to the respiratory epithelium. Little is known about the recovery and regeneration of the epithelium following COVID-19. Poor recovery could leave individuals at risk of secondary bacterial infection and persistent symptoms.

The aim of this study was to assess epithelial recovery following SARS-CoV2 infection across a range of acute illness severities.

Methods 41 people were recruited at 3-12 months post PCR confirmed SARS-CoV2 infection. The respiratory epithelium was sampled by brushing the nasal inferior turbinate. Ciliary function was assessed by high-speed video microscopy and ultrastructure was assessed by electron microscopy. A subset of patients had repeat nasal brushing 3-5 months following their first visit. Demographics, severity of infection and longitudinal symptoms were recorded for comparison. Results were compared to healthy controls and historical controls recruited prior to the pandemic.

Results Post-COVID epithelium was friable and most samples contained detached single cells and blood. Epithelial disruption 